\title{
The Human Organism is a Biophysical-Biopsychological System
}

\author{
Janos Vincze, Gabriella Vincze-Tiszay \\ Health Human International Environment Foundation, Budapest, Hungary \\ e-mail: ndp@t-online.hu
}

\begin{abstract}
Healthy and ill people both try to achieve the required performance and satisfaction of this, but they experience differently their activities accompanied by secondary feelings, their critical attitude and their compliance with social desires. Exceeding these correspondences requires the operation of countermeasures in all people, which at times are barely noticeable because they are embedded in the personality structure or alternately use the entire arsenal of mechanisms. If a biopsychological system responds specifically to an external stimulus, it means that the value of some of its parameters changes and then returns to its original state of homeostasis when the response is complete. Parameters characterized by static equilibrium and is involved in the response return their values over time to their original value such that its envelope is an exponential (ascending or descending) curve. The parameter that is characterized by a state of dynamic equilibrium and is involved in the response returns to its original value with attenuated harmonic oscillation after its completion. The duration of the return is different for each parameter, and the normal state of homeostasis is determined by the parameter with the slowest finish.
\end{abstract}

Key words: biophysical modelling, system, pathomechanism, somatopsychic-, psychosomatic-specificity

\section{Introduction}

Biophysical modeling is a method that can be used to study biological mechanisms - that is, it involves mathematical analysis and model systems. The models serve as analogues of operation and regulation, based on information signals specific to the living organism, and thus monitor their mechanism of operation until different automatic regulations of the normal state and different, that is deregulated functions and complexity. [1]

The deregulated state can be followed not only as an internal functioning of the body, but also as a mechanism for influencing external influences, which gives rise to new assumptions and other points of departure, that help model autoregulatory systems. The principle of autoregulation is that the operation of the system and the transmission and conversion of its energy quantities are controlled by the subsystems. The sub-units, as units, provide information on the operation to be performed, and provide feedback on these sub-results by providing signals, that include both new and old status or status information. [2]

The homeostasis of the human body affects not only internal regulation but also thinking and behavior. Homeostasis is all a system property, being served by the harmonious and unified integrity of the entire body. The emergence of a behavior is the consequence of a change in the internal or external environment; the response assumes psycho-organic coordinated co-operation of the whole organism. [3]

Escaping into the role of a sick person is when experiencing a conflict creates a psychological symptom. This mental illness enables one to endure conflict by changing the social role of the 
individual as they escape to sickness. The secondary benefit of the escape to illness is the delay of resolving conflict. This is the phenomenon of conversion.

\section{The system}

The environment $\mathrm{K}$ of the system $\mathrm{R}$ is what is outside the system. Everything that does not belong to the given system is called environment. This is also not easy to define. [4] This is why the scientist must have considerations of the system environment that are richer and more complex than the mere study of boundaries P (edge). Very often, the environment is not only beyond the control of the system, but also something that partly determines how the system works.

$$
\mathrm{P}=\mathrm{R} \cap \mathrm{K}
$$

The system and environment are not disjointed. What they have in common is the edge of the system, the finite-sized closed surface that separates the system from the environment. Through the edge, there is an interaction between the system and the environment. [5]

The edge is part of both the system and its environment. Its position determines the static location of the system, its geometric location within the environment. At the same time, however, as a medium that can be characterized by a factor of conductivity, it determines the dynamic relationship between the system and the environment, as well as the interactions between them. [6]

In order for a system to become the subject of scientific study, it must be precisely separated from its environment in space and time. This is only possible if, on the one hand, we are able to highlight the system in accordance with the established interconnection rules, and if, on the other hand, we are able to delineate the relationships and interactions between the system and its environment. In this case, the subsystems appear inside the system on each other or on the system, or the coercive effect of the system on one or the other, which is also influenced by the edge conditions, through their so-called coefficients of conductivity $\left(\mathrm{V}_{\mathrm{jk}}\right)$.

The numerical value of the coefficient of conductivity gives the current of the extensive quantity (w) for a unit time difference of an intensive quantity $(\mathrm{z})$. If the edge is divided into $\mathrm{S}_{\mathrm{k}}$ units of homogeneous surface:

$$
\mathrm{P}=\sum \mathrm{S}_{\mathrm{k}}
$$

Then the total flow of the extensive quantity $\mathrm{w}_{\mathrm{j}}$ over the surface of the whole edge $S$ :

$$
\mathbf{w}_{\mathbf{j}}=\iint_{S} S_{k} \sum_{r=1}^{n} v_{j r} \operatorname{grad} z_{r}
$$

This seemingly complicated formula becomes easier to understand when we think of the cells that make up living organisms. [7] Conductivity indicates that the cell membrane is permeable to a particular substance. The permeability of a surface also varies with time per unit. If impermeable to a given material, then the row vector of the conductivity matrix consists of zeros. If we apply it to the vectors of the conductivity matrix column, it means the effect a generalized force $F$ has on the corresponding flux $\varphi$.

Systems can also be characterized by their function:

- keeping the system characteristic $\mathrm{G}_{\mathrm{i}}{ }^{*}$ at the prescribed value;

- keeping the output at the specified value $/ \mathrm{o}^{*}(\mathrm{t}) /$;

- safe input-output / $\mathrm{i}(\mathrm{t}) \rightarrow \mathrm{o}(\mathrm{t}) /$ conversion.

These three functions may change over time.

The four essential features of living systems are, in short, the set of content components, structural construction, communication, and intermittent parameters that implement the functions. (Table. 1.) If we provide a definition of transport phenomena in living organisms and its mathematical description, it would result in the elaboration of the interaction between the system and its environment for the relevant specific case. By transport phenomena we mean the change in "generalized forces" in time and space when they create fluxes to which the conservation laws apply. [8] 
Table1. A possible classification of systems

\begin{tabular}{|c|c|}
\hline Aspect & Version \\
\hline Task & Best-first \\
& search \\
& Regulation \\
\hline $\begin{array}{c}\text { Functioning } \\
\text { mode }\end{array}$ & $\begin{array}{c}\text { Discontinuous } \\
\text { Continuous }\end{array}$ \\
\hline Behavior over & Constant \\
time & Varying \\
\hline Property of & Deterministic \\
signs & Stochastic \\
\hline Character & Linear \\
& Non-linear \\
\hline Internal & Constant \\
structure & Adaptive \\
& Learning \\
& Self-guided \\
\hline
\end{tabular}

According to the degree of integrity, systems are usually divided into two major groups: integral and summative systems. Integral are systems the subsystems of which are not equivalent and in which the removal of some subsystems may result in the disintegration of the system. In integrative systems (such systems are living systems) there is a degree of generalized regulation that results from integrity itself. [9] The feedback principle known from cybernetics does not apply to this regulation. Summative systems are systems of equivalent elements in which the removal of individual elements does not significantly change the properties of the system. In connection to this division, we must emphasize its relative nature.

The summative system, due to the external nature of the relationships, does not have its own constancy that is relatively independent of the state of the constituent elements; the ability to repair potentially damaged interactions is lacking. These systems can be represented by the following type of connection, according to Klaus:

$$
\mathbf{R}(\mathrm{x}, \mathrm{y}) \rightarrow \mathbf{R}^{\prime}\left(\mathrm{x}^{\prime}, \mathrm{y}^{\prime}\right) \quad\left\{\begin{array}{l}
\mathbf{x}^{\prime}=1 . \mathrm{x} \\
\mathbf{y}^{\prime}=2 . \mathrm{y}
\end{array}\right.
$$

Integrated systems are established on the basis of internal, mutually compatible connections. In a generalized form, the integral system can be represented in the form of the following structurally different relationship from the previous one:

$$
R(x, y) \rightarrow R^{\prime}\left(x^{\prime}, y^{\prime}\right) \quad\left\{\begin{array}{l}
x^{\prime}=2 . x-y \\
y^{\prime}=3 . x-2 . y
\end{array}\right.
$$

This relationship represents the correlative dependence of the two component variables ( $\mathbf{x}$ and $\mathbf{y})$ within the global system R: $\mathbf{x}$ ' varies not only according to $\mathbf{x}$ but also $\mathbf{y}$, just as $\mathbf{y}^{\prime}$ varies not according to $\mathbf{y}$ but also $\mathbf{x}$. More specifically, in an integral system, a change in the state of one subsystem also affects the state of the other subsystems, and vice versa. This is of great importance in the implementation of regulations within the system. 


\section{The human organism is as adjustment system}

The human body behaves like a morphofunctional unit in the continuous process of adaptation to the environment. The organism's unity is not given by its homogeneity, but on the contrary, by its highest heterogeneity. From the sub-cellular structural-functional levels, step after step, to the ultimate level - the organism - heterogeneity amplifies, which requires more and more complex adjustment mechanisms, with more and more complex interrelations. [10]

By adjustment we understand the process through which a size is continuously measured and, by comparison with another reference value, it is influenced in the meaning of an equalization. This way, the regulated unit is based on two components: comparison and command.

Adjustment, with reference to the human body aims, within the biologically optimal limits of the structure and functionality, to all the steps of organization: maintaining the homeostasis of the internal environment, coordinated and unitary functionality of all its components, the adaptation of functionality to the requirements imposed by the environment. The adjustment also has an organization role in the growth and development of the organism, as well as in its reproduction. The organism is a system ensemble situated in a dynamic equilibrium permanently controlled through the adjustment loops. [11]

The optimal systems ensure the coincidence between the regulated size and the reference one through negative retroaction, compensating the perturbation. Extreme adjustment or automatic optimization systems also have a retroaction loop, but there is no comparison between the regulated size and a reference size, hence, by seeking for the extreme point, they elaborate adequate orders for maintaining the biophysical and physiological process in that point.

Within the reverse connection, we can distinguish two fundamental forms of this mechanism: negative inverse connection and the positive one. According to the classical definition we name negative reverse connection the mechanism through which, after the comparison of the value of the exit signal with the desired value, the adjustment initializes a reverse process in relationship with the deviation direction. [12]

The feed-back or negative inverse connection functions "constantly" and its main role is, to maintain certain functional parameters of the body to a relatively constant value. This is about a relative stability, since the adjustment involves oscillations around an abstract medium value with a tendency to minimize the deviation in respect of this mean value.

Let's denote with $\mathbf{o}(\mathbf{t})$ the exit output and the mean value of the characteristic parameter on the system is $\mathbf{o}^{*}(\mathbf{t})$; after the adjustment, the values of the outputs obtained shall be denoted with

$$
\begin{gathered}
o\left(t_{1}\right), o\left(t_{2}\right) o\left(t_{3}\right), \ldots, o\left(t_{n}\right)=o^{*}(t) ; \\
\quad \text { if } \quad t_{1}<t_{2}<t_{3}<\ldots<t_{n} .
\end{gathered}
$$

We talk about a negative inverse value, if the following two conditions are satisfied:

$$
\begin{gathered}
\left|\mathbf{o}^{*}(\mathbf{t})-\mathbf{o}\left(\mathbf{t}_{1}\right)\right|>\left|\mathbf{o}^{*}(\mathbf{t})-\mathbf{o}\left(\mathbf{t}_{2}\right)\right|>\ldots>\left|\mathbf{o}^{*}(\mathbf{t})-\mathbf{o}\left(\mathbf{t}_{\mathbf{n}}\right)\right| \\
\frac{d\left|o\left(t_{i}\right)-o^{*}(t)\right|}{d t} \leq 0
\end{gathered}
$$

In the living organisms, all the mechanisms for maintaining a constant composition and internal environment function on the basis of on negative feedback. 
We call "reverse positive connection", the phenomenon in which the value of the exit system differs from the desired value and the adopted decision will stress this difference. Hence, the reverse positive connection produces an increase of the value of the exit signal if it were higher, respectively a decrease of the exit value if it were lower than the desired value.

The positive reverse connection is the connection which introduces an amplification of the effect caused by the entry signals. Maintaining the denotations above, we speak about a reverse positive connection, if it fulfils the following two conditions:

$$
\begin{aligned}
\left|\mathbf{o}^{*}(\mathbf{t})-\mathbf{o}\left(\mathbf{t}_{1}\right)\right| & <\left|\mathbf{o}^{*}(\mathbf{t})-\mathbf{o}\left(\mathbf{t}_{2}\right)\right|<\ldots<\left|\mathbf{o}^{*}(\mathbf{t})-\mathbf{o}\left(\mathbf{t}_{\mathbf{n}}\right)\right| \\
\frac{d\left|o\left(t_{i}\right)-o^{*}(t)\right|}{d t} & \geq 0
\end{aligned}
$$

\section{The biopsychological system}

"Mens sana in corporis sano" - that is "a healthy mind in a healthy body" - refers to the inseparable unity of the biological and the psychic. Neither can exist without the other. No matter how elusive psychic events are, the organic substrate for this is provided by our individual biological structure. The unit is thus made up of two parts, operating separately, but mutually determining each other's functionality. An equilibrium system develops in which when a function is disrupted it burdens the functioning of the organs associated with it which creates a dysfunction in the organ itself as well as in the body and they react to each other. For example, in the case of anorexia or bulimia, both of which have psychological causes and are somatic, the nerve center responsible for disrupted hunger and satiety generates organic damage.

In this, from a biophysical point of view, there are psychosomatic and somatopsychic, i.e. psychiatric disorders caused by organic dysfunction (the latter, for example, kidney diseases, brain tumors, gastric ulcers, etc. are accompanied by psychological changes) are formed. Emotional stress results in anxiety with vegetative accompanying phenomena (sweating, temperature changes, flushing-paleness, nausea, diarrhea, urinary urgency, etc.). If these vegetative phenomena are repeated, they can also result in organ damage. This is called psychosomatic specificity. Psychic emotion can also affect the effects of certain hormones, not just the functioning of certain organs. A significant proportion of psychosomatic disorders are reactions that persist in the normal state, and only become prolonged and increase in intensity. Repeated stimulus responses result in a qualitative jump from quantity after a period of time, which can lead to a greater or lesser degree of temporal or irreversible changes. A state of an endless cycle is created in the closed system, which thus requires external intervention because the balancing role of homeostasis is disrupted. Based on all this, different groupings can be established: primary and secondary triggers or causes, a symptom of a neurotic or organic process that occurs or develops as a result of an endogenous or exogenous effect, the internal pathomechanism of which must be interfered with. [13]

The highest level of expression of the operational polyvalence of the human psyche is considered to be creative activity. The distance between reality and model, aspiration and opportunity is an indicator that determines a person's actual level of self-realization.

Since these indicators define the whole of psychic organization, they can be used to assess the general level of development of a personality.

The level of organization, which expresses the correspondence of behavior to the nature and meaning of external influences, can be estimated by constructing a phase profile of the psychic system based on the main psychic indicators.

This can be expressed in the form of the following relation: 


$$
S=\frac{\sum_{j=1}^{m} Y_{j}\left(t_{k}\right)}{\sum_{i=1}^{n} X_{i}\left(t_{k}\right)} ; k=1,2, \ldots, p
$$

where $\sum_{j=1}^{m} Y_{j}\left(t_{k}\right)$ is the sum of the mean of the adaptive responses and $\sum_{i=1}^{n} X_{i}\left(t_{k}\right)$ is the sum of the external effects and stresses, $t_{k}(k=1,2, \ldots, p)$ expresses the consecutive motions of the examined period. If the value of $S$ is close to zero, then the personality is characterized by a great lack of psychic organization, i.e. psychic disorganization; if the value of $S$ is close to 1 , the individual is expected to respond correctly at all times, to show a state of psychological equilibrium, and to have minimal psychological fluctuations. [14]

With the technical advances that have taken place in our environment over the last hundred years, our body's usual biological adaptation has not been able to keep up with evolution, and as a shield against the emotional problems that have arisen, it has hidden behind psychosomatic perturbations. Personality developmental disorders can occur as a result of both internal (endogenous) and external (exogenous) influences, due to the disharmony of this co-operation, some personal characteristics become disproportionately predominant, others become irrelevant. If an individual's responses come from an external cause, mostly reversible processes appear. The triggering causal factors are correlated in time and intensity, i.e. they occur during abnormally strong and prolonged exposure and are usually in direct proportion to the strength of the effect and the severity of the symptom. As emotional order and cognitive tension decrease, the reaction slowly dissolves.

\section{Epilogue}

By examining the life disappears as we are approaching the lifeless components from the total life. That is, life doesn't equal with the sum of its constituents!

The better we disconnect these living units the farther we are gradually distancing ourselves from the biology, then arriving to the superb, eternal and universally physical laws of the lifeless material. The living material is a highly organized complex structural system, able to maintain this composite structure through its metabolism. It is proper to ascribe the qualitative differences within the single internetics levels to the organization of the constituents. It is not possible to understand the totality through its particles, however, the knowledge of the parts would help us to understand the whole.

\section{References:}

[1] Vincze, J. 2007. Interdisciplinarity, NDP P., Budapest.

[2] Vincze, J. 2015. The Biophysics is a Boderland Science. Second Ed. NDP P., Budapest.

[3] Vincze, J.: 2018. Medical Biophysics. NDP P., Budapest.

[4] Bertalanffy, Ludwig von 1968. General System Theory - Foundations, Development, Applications; Revised edition, George Braziller, New York.

[5] Marshall, A. G. 1978. Biophysical Cjemistry: Principles, Techniques and Applications. John Wiley and Sons.

[6] Dutreix, J., Desgrez, A., Bok, B., Chevalier, C. 1973. Physics and Biophysics. (in French: Physique et Biophysique.) Masson, Paris.

[7] Donskoi, D., D. 1975. Gundlagen der Biomechanik. Sportverlag, Berlin.

[8] Vincze,J.: 2020. The Biophysics of the Human Apparatuses. NDP P., Budapest.

[9] Grémy F., Pagèt, S. F. 1966. The Elements of the Biophysics. (in French: Eléments de biophysique.) Ed. Med. Flammarion, Paris.

[10] Vincze, J., Vincze-Tiszay, G. (2019). The "hypothetical secondary brain", Medical Research Archives. 7(7):1-3. 
[11] Vincze, J. Vincze-Tiszay, G. (2020). The Biophysical Modeling of the different Regulations in the Human Organism. Intern. J. Inovat. Studies Med. Sciences, 4(1):1-4.

[12] Vincze J., Vincze-Tiszay, G. (2020). The Biophysical Adjustment in the Human Organism. J. Med. Res. Case Report,2(3)1-7.

[13] Vincze, J., Vincze-Tiszay, G. (2020). The Biophysics is a Borderland Science. Roum. J. Appl. Scien. Tech. (2), 3:115-121.

[14] Vincze, J. (2020). The Biophysical Modeling of the Apparatuses in the Human Organism. Lambert Academic Publishing, Berlin. 